Check for updates

Cite this: RSC Adv., 2017, 7, 37898

Received 14th April 2017

Accepted 19th July 2017

DOI: $10.1039 / c 7 r a 04243 d$

rsc.li/rsc-advances

\section{An electrochemical sensing platform for trace recognition and detection of an anti-prostate cancer drug flutamide in biological samples $\dagger$}

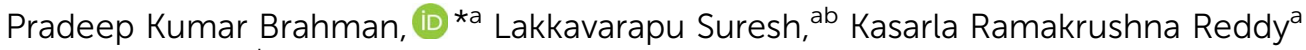 \\ and Bondili J. S.
}

Developing simple, selective and sensitive platforms for anticancer drug analysis is of great importance in biomedical research. This study describes the fabrication of a ferrocene (FC) and multiwalled carbon nanotubes (MWCNTs) functionalized carbon paste electrode as a highly sensitive electrochemical sensor for the determination of anticancer drug flutamide in serum, plasma and urine samples. Scanning electron microscopy (SEM), cyclic voltammetry (CV), electrochemical impedance spectroscopy (EIS) and chronocoulometry methods were used to characterize the sensor. Electrochemical impedance studies revealed that the smaller $R_{\mathrm{ct}}$ (charge transfer resistance) value was observed at FC/MWCNTs/CPE as compared to that of MWCNTs/CPE and CPE, which authenticates its good conductivity. The obtained sensor has a large specific area, high conductivity, good biocompatibility and fast redox properties. Various optimization parameters such as $\mathrm{pH}$, accumulation time, deposition potential, scan rate and the effect of composite mixture on the electrochemical performance of the sensor were evaluated. A linear range from 0.1 to $110 \mu \mathrm{M}$ for FT detection was obtained with a detection limit of $0.001 \mu \mathrm{M}$. Furthermore, the fabricated sensor has been utilized for estimation of FT in biological samples. This efficient strategy provides new insight into the design of novel flexible sensors for a wide range of applications in biosensing, bioelectronics, and clinical applications.

\section{Introduction}

Among all anticancer drugs flutamide (FT), 4-nitro-3-trifluoromethyl-isobutilanilide (Scheme 1), is the primary nonsteroidal anti-androgen drug used to treat prostate cancer. ${ }^{\mathbf{1}}$ Its action is based on blocking the effect of the male hormone, testosterone, whose supply is essential for the growth of prostate cancers. This drug and its primary hydroxy metabolite decrease metabolism of C-19 steroids by the cytochrome P-450 system at the target cells in the secondary sex organ. ${ }^{2}$ The prostate cancer cells are unable to grow without testosterone. FT does not cure prostate cancer, but it can keep the cancer under control for many months or years.

During the past two decades great efforts have been made towards improving molecular sensing of chemically and biologically species. ${ }^{3-10}$ Electrochemical sensors have been proven as an inexpensive, simple and versatile analytical tool with remarkable detection sensitivity, reproducibility, ease of

${ }^{a}$ Electroanalytical Lab, Department of Chemistry, $K$ L University, Guntur, Andhra Pradesh, India. E-mail: tiwari25_pradeep@yahoo.com; drpkt@kluniversity.in; Fax: +91-8842-547546; Tel: +91-9407-592987

${ }^{b}$ Department of Biotechnology, K L University, Guntur, Andhra Pradesh, India

$\dagger$ Electronic supplementary information (ESI) available. See DOI: 10.1039/c7ra04243d miniaturization and specific selectivity of target molecules. ${ }^{\mathbf{1 1 - 1 8}}$ They have found a vast range of important applications in the fields of clinical, industrial, environmental and agricultural analyses. ${ }^{19-21}$ In addition the modification of electrode surface to act as a sensor is a widely used electroanalytical strategy useful for many sensing applications requiring highly compatible and properly functionalized surfaces to anchor the recognition material. However, development of a biosensor with improved parameters such as selectivity and stability, which depend on the biosensing molecule attachment and its compatibility with the solid surface, requires extensive investigation. Carbon nanotubes (CNTs) have been considered as a promising material for the development of biosensor with enhanced sensitivity because of its high chemical stability, good<smiles>CC(C)C(=O)NC1=CC(C(F)(F)F)C([N+](=O)[O-])=CC1</smiles>

Scheme 1 Chemical Structure of flutamide. 
electrical conductivity, and tubular structure..$^{22-26}$ Moreover, the high conductivity and charge transfer channels of CNTs make them a promising material for composite fabrication applications. In recent years, MWCNTs have been one of the most intensively investigated nanostructured materials. ${ }^{27-33}$ Chemical functionalization of MWCNTs can enhance specific surface area and thereby improve electrical conductivity, mechanical properties and compatibility for immobilization of biomolecules.

Over the past two decades, chemically modified carbon paste electrodes $^{34}$ (CMCPEs) have attracted broad interest in biological and pharmaceutical sensing development due to low background current, wide range of potential window, easy surface renewal, lower detection limit, and low cost. Due to these advantages CMCPEs have been employed for several purposes, such as voltammetric determination of the herbicide propham, ${ }^{35}$ electroanalytical determination of 4-chloro-3methylphenol, ${ }^{36}$ electrochemical analysis of $\mathrm{D}$-penicillamine (DPA), stripping measurements of hydrogen peroxide, electrocatalytic determination of ascorbic acid etc. ${ }^{37-39}$ An electroactive mediator needs to be of low relative molar mass, reversible, fast reacting, regenerated at low potential, $\mathrm{pH}$ independent, stable in both oxidized and reduced forms, unreactive with oxygen and non-toxic. Among the most successful mediators are those based on ferrocene and its derivatives, all of which meet the above criteria.

Drug analysis, which is an important branch of analytical chemistry, plays an important role in drug quality control. Many analytical methods for the determination of FT in biological fluids and in pharmaceutical preparations are reported in the literature. These methods include, high-performance liquid chromatography (HPLC), ${ }^{40}$ spectrophotometry ${ }^{\mathbf{4 1 4 2}}$ and gas chromatography ${ }^{\mathbf{4 3}}$ etc. Since, some of these methods are timeconsuming, expensive or need complicated pretreatment, such as multi solvent extraction, as well as trained technicians to carry out the measurements, which limit their applications. However, because it is an electroactive compound, FT can be detected by electrochemical methods. This is advantageous because such detection is simple, sensitive, selective, responsive, and inexpensive. Therefore, little bit voltammetric study has been carried out for the electrochemical detection of FT. ${ }^{\mathbf{4 4 , 4 5}}$ To the best our knowledge no report is available in the literature for the detection of flutamide based on FC and MWCNTs modified carbon paste electrode.

The present study deals with the fabrication of a novel electrochemical sensor based on FC and MWCNTs functionalized carbon paste electrode. The electrochemical behaviour of FC/MWCNTs/CPE was investigated to evaluate of its suitability for the detection and analysis of FT using square wave voltammetry. The surface properties of the FC/MWCNTs/CPE allow FT to be adsorbed effectively onto the fabricated sensor. This improves the sensitivity of the sensor such that it can be used for the detection of FT in biological samples. We have optimized the experimental parameters and developed a simple, stable, and sensitive method for the detection of FT. To the best of our knowledge, the combination of FC and MWCNTs modified CPE for the detection and quantification of FT has not been explored so far. The fabricated sensor has displayed fast electron transfer and prominent catalytic ability towards FT with wide linear range, low detection limit and large surface concentration. Therefore, this work opens a new channel to the construction of highly sensitive sensors by employing composite material containing FC and MWCNTs.

\section{Experimental}

\subsection{Chemicals and reagents}

Acetic acid, sodium acetate, MWCNTs (99\%), paraffin oil obtained from Himedia, India. Graphite powder, flutamide, $\mathrm{KH}_{2} \mathrm{PO}_{4}, \mathrm{~K}_{2} \mathrm{HPO}_{4}$ Tris buffer and ferrocene was procured from sigma, India. All the reagents were of analytical graded and used without further purification. All required solutions were prepared using double distilled water.

\subsection{Instrumentation}

The voltammetric measurements were performed using an Autolab PGSTAT 204 equipped with USB electrochemical interface using NOVA software. Conventional three-electrode system employing, a CPE (unmodified and modified) was used as working electrode, platinum wire, and $\mathrm{Ag} / \mathrm{AgCl}$ (sat. $\mathrm{KCl})$ were used as counter and reference electrodes, respectively. The $\mathrm{pH}$ measurements were performed using systronic$362 \mu \mathrm{pH}$ meter. Morphological analysis was carried out using SEM (FEI INSPECT F50).

\subsection{Preparation of CPE and FC/MWCNTs/CPE}

The CPE was prepared by thoroughly mixing fine graphite powder with mineral oil at composition $70: 30$ (w/w graphite/ oil) using a motor and pestle and was allowed to homogenize for 24 hours. FC/MWCNTs/CPE was prepared by mixing mineral oil, graphite powder and ferrocene with various weight ratios. A portion of the paste was then packed into a Teflon micro tip (diameter $2.0 \mathrm{~mm}$ ) and a copper wire was inserted to establish an electrical contact. Electrode surface was smoothed on a zero grade butter paper to produce a reproducible working surface. For comparison MWCNTs/CPE was also prepared by mixing graphite powder, mineral oil and MWCNTs at composition $60: 30: 10(\mathrm{w} / \mathrm{w})$.

\subsection{Voltammetric study}

Cyclic voltammetric (CV) and square wave voltammetric (SWV) studies were carried out for FT analysis. Stock solution of FT was prepared in $90 \%$ ethanol. An appropriate aliquot was taken in $50 \mathrm{ml}$ standard volumetric flask and made up to the mark with $0.2 \mathrm{M}$ acetate buffer ( $\mathrm{pH}$ 4.5). The solution was then transferred into an electrochemical cell and the measurements were carried out at $25{ }^{\circ} \mathrm{C}$. The $\mathrm{CV}$ and SWV studies were carried out by sweeping the potential between 0.0 to $-1.0 \mathrm{~V}$. The voltammograms were recorded using unmodified and modified carbon paste electrodes. The EIS studies were performed on $5 \mathrm{mM}$ $\left[\mathrm{Fe}(\mathrm{CN})_{6}\right]^{3-} /\left[\mathrm{Fe}(\mathrm{CN})_{6}\right]^{4-}$ in $0.1 \mathrm{M} \mathrm{KCl}$ in the frequency range from $10^{-1}$ to $10^{5} \mathrm{~Hz}$ at open circuit potential with amplitude of $5 \mathrm{mV}$. 


\subsection{Preparation of solution for the determination of FT in} biological samples

Urine and blood serum samples were obtained from local pathology laboratory Sri Diagnostic Centre and all ethical guidelines were followed in handling the samples for all measurements and ethics approval for the use of human subjects was obtained from Institutional ethics committee. $0.2 \mathrm{ml}$ blood (serum and plasma)/urine sample was taken in a voltammetric cell and made up to $40 \mathrm{ml}$ with acetate buffer. This solution was spiked with the known volume of standard drug sample. Calibration method was employed for quantification of FT in all serum, plasma and urine samples.

\section{Results and discussion}

\subsection{Morphological characterization of fabrication sensor}

The morphology of the FC/MWCNTs/CPE sensor was studied by SEM. Fig. 1 shows the SEM images of CPE, MWCNTs/CPE and FC/MWCNTs/CPE. The comparison of SEM images shows a difference in the morphology of all three electrodes. The unmodified CPE surface is homogenous and a layer of irregular flakes of graphite powder is present and isolated from each other (Fig. 1A). The SEM of CPE modified with MWCNTs was characterized by presence of small bundles like structure (Fig. 1B). Also, it can be obviously seen that the FC dispersed successfully in the MWCNTs/CPE electrode (Fig. 1C).

\subsection{Characterization of fabricated electrochemical sensor by cyclic voltammetry}

Cyclic voltammetry (CV) was used to elucidate the sensitivity and electrochemical properties of FC/MWCNTs/CPE sensor. For this purpose, different electrodes namely CPE, MWCNTs/CPE and FC/MWCNTs/CPE have been compared using $15 \mu \mathrm{M}$ FT in acetate buffer solution $\mathrm{pH}$ 4.5. Potential range was applied for this study is 0.0 to $-1.0 \mathrm{~V}$. CV plots for $15 \mu \mathrm{M}$ FT at all three electrodes are depicted in Fig. 1D. It can be seen in Fig. 1D, that FC/MWCNTs/CPE exhibits higher peak currents for the reduction of FT in comparison with the other electrodes, which can be related to the high surface area and electrocatalytic behaviour of MWCNTs and FC. In comparison with the other electrodes, FT displayed better electrochemical behavior at the FC/ MWCNTs/CPE, which indicated that fabricated sensor provided the conducting bridges in order to promote the electron transfer of FT. Fig. 2A shows the influence of scan rate on reduction peak current and peak potential of $15 \mu \mathrm{M}$ FT. It was observed that
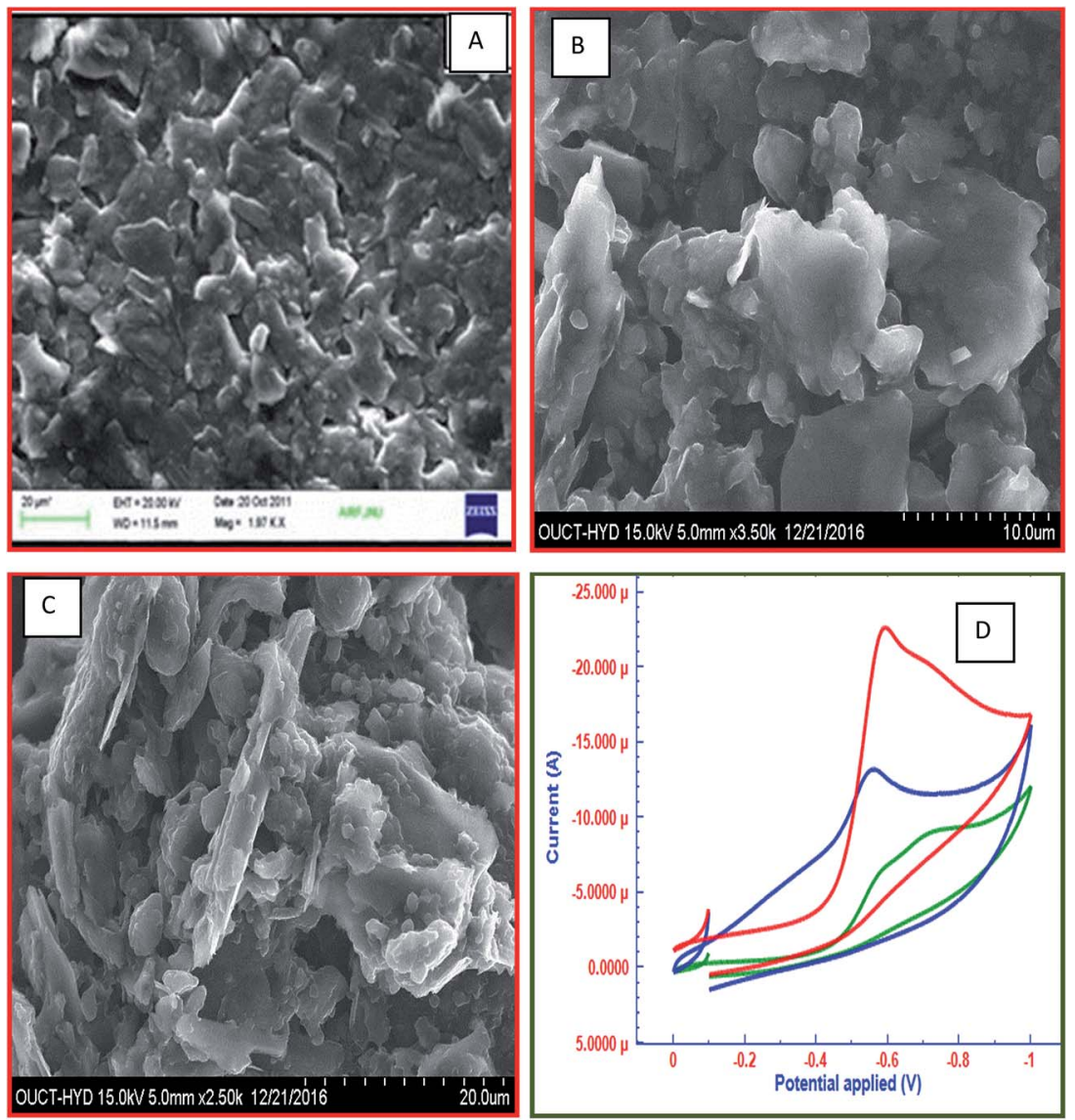

Fig. 1 (A-C) SEM images of CPE, MWCNTS/CPE and FC/MWCNTs/CPE, (D) cyclic voltammograms obtained for $15 \mu \mathrm{M}$ FT at three different electrodes: CPE (—), MWCNTs/CPE (-) and FC/MWCNTs/CPE (-), voltammetric conditions: scan rate $50 \mathrm{mV} \mathrm{s}^{-1}$, scanning potential range between 0.0 and $-1.0 \mathrm{~V}$, supporting electrolyte $(0.2 \mathrm{M})$ acetate buffer $\mathrm{pH} 4.5 ; n=3$. 

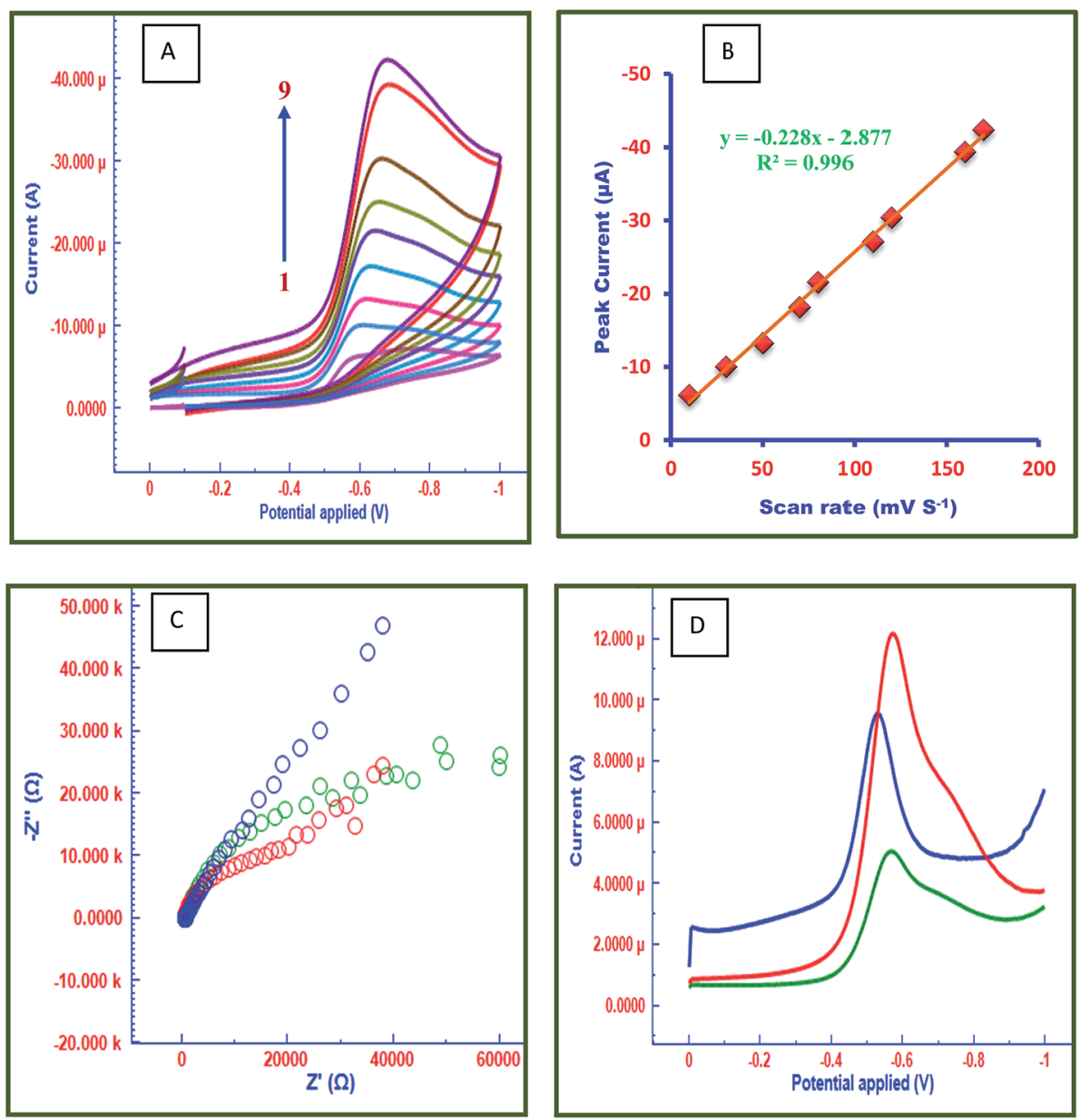

Fig. 2 (A) Cyclic voltammograms of $15 \mu \mathrm{M} \mathrm{FT}$ at different scan rates $\left(1-9 \mathrm{mV} \mathrm{s}^{-1}\right.$ ): 10, 30, 50,70, 90, 110, 120, 160 and 170; (B) /p vs. Scan rate plot for the data obtained from (C); (D) Nyquist plots for EIS measurements $\left(5 \mathrm{mM}\left[\mathrm{Fe}(\mathrm{CN})_{6}\right]^{3-} /\left[\mathrm{Fe}(\mathrm{CN})_{6}\right]^{4-}\right)$ at three different electrodes: $\mathrm{CPE}(0000)$, MWCNTS/CPE (0000) and FC/MWCNTs/CPE (0000), (D) square wave voltammograms of $15 \mu \mathrm{M}$ FT at three different electrodes: CPE (—), MWCNTs/CPE $(\longrightarrow)$ and FC/MWCNTs/CPE ( $(-)$, voltammetric conditions: $E_{\mathrm{acc}}=0.1 \mathrm{~V}, t_{\mathrm{acc}}=120 \mathrm{~s}$ in acetate buffer pH 4.5 , step potential $=$ $5 \mathrm{mV}$ and modulation potential $=50 \mathrm{mV}$.

reduction peak current increased linearly with increasing scan rate ranging from $10 \mathrm{mV} \mathrm{s}^{-1}$ to $170 \mathrm{mV} \mathrm{s}^{-1}$ (Fig. 2B).

The number of electrons $(n)$ involved in the reaction was calculated from the cyclic voltammetry. $E_{\mathrm{p}}-E_{\mathrm{p} / 2}$ value was calculated to be $23.7 \mathrm{mV}$. This value was then substituted in the following equation to obtain ' $n$ ' value:

$$
E_{\mathrm{p}}-E_{\mathrm{p} / 2}=(47.7 / \alpha n) \mathrm{mV}
$$

Solving this eqn (1), the $\alpha$ na value is found to be 2.01. Now, for a totally irreversible reaction, the electron transfer coefficient $(\alpha)$ is assumed to be 0.5 . Therefore, by substitution of the value of $\alpha$ in the above equation provides the value of $n=4$ for the reduction of FT. Following expected reduction mechanism of FT can be predicted (Scheme 2).

\subsection{Electrochemical impedance spectroscopy}

Electrochemical impedance spectroscopy (EIS) was used as an efficient tool to characterize the interface properties of the electrode surfaces. ${ }^{46}$ The EIS can give information on the impedance changes of the electrode surface during the modification process. The EIS includes a semicircular part and a linear part. The semicircular part at higher frequencies corresponds to the electron transfer limited process and the diameter is equivalent to the electron transfer resistance $\left(R_{\mathrm{ct}}\right)$. The linear part at lower frequencies corresponds to the diffusion process. Fig. 2C presents the Nyquist diagrams of CPE, MWCNTs/CPE and FC/ MWCNTs/CPE in $5 \mathrm{mM}\left[\mathrm{Fe}(\mathrm{CN})_{6}\right]^{3-} /\left[\mathrm{Fe}(\mathrm{CN})_{6}\right]^{4-}$ at an applied voltage of $5 \mathrm{mV}$ in the frequency range from $10^{-1}$ to $10^{5} \mathrm{~Hz}$. The frequency range selected in the experimental studies was from $10^{-1}$ to $10^{5} \mathrm{~Hz}$. The EIS of the CPE and MWCNTs/CPE exhibit a semicircle that shows a much higher interfacial electron transfer resistance. Whereas the EIS of the FC/MWCNTs/CPE film are close to a line, showing very low electron transfer resistance due to the large surface area, excellent electrocatalytic nature and high adsorption tendency of FC/MWCNTs/CPE. The charge transfer resistance $\left(R_{\mathrm{ct}}\right)$ obtained for CPE, MWCNTs/CPE and FC/MWCNTs/CPE are $3.512 \mathrm{kohm}, 2.235 \mathrm{kohm}$ and 0.531 kohm, respectively. Therefore, this fabricated electrode was used for the electrochemical determination of FT. 

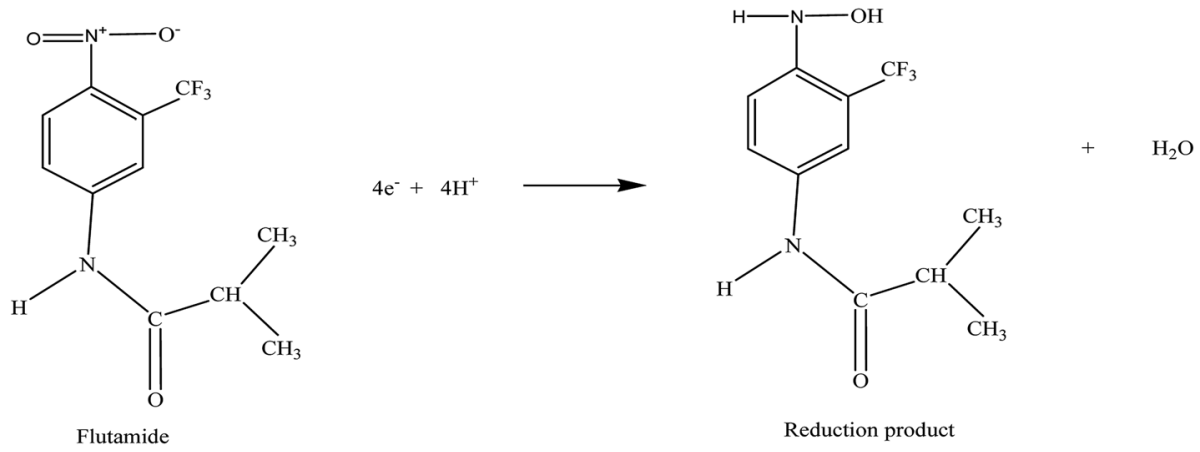

Scheme 2 Proposed mechanism of electro-reduction of flutamide at fabricated sensor.

\subsection{Chronocoulometry}

Chronocoulometry was employed for the determination of the kinetics, and mechanism of electrode reactions involved in the electro-oxidation of $15 \mu \mathrm{M}$ FT at CPE, MWCNTs/CPE and FC/ MWCNTs/CPE. The diffusion coefficient and $Q_{\text {ads }}$ of FT were calculated from the plot of $Q v s . t^{1 / 2}$ using Anson equation. The resulting calculated parameters are presented in Table 1. As can be seen from the table, the value of the slope and the $Q_{\text {ads }}$ for the FC/MWCNTs/CPE were more than that for other electrodes, confirming the more accumulation of FT onto the electrode surface more effectively (Table 1 ).

The surface coverage $\left(\Gamma^{0}\right)$ for all three electrodes was calculated using the following relationship:

$$
Q_{\mathrm{ads}}=n F A \Gamma^{0}
$$

and the results are presented in Table 1 . Here $F$ is Faraday's constant and $A$ is electrode surface area. From these values, it was observed that the surface coverage was maximum in the case of the FC/MWCNTs/CPE. Thus, due to the synergistic effect of FC and MWCNTs the electrode surface coverage by FT drastically increased and the kinetics of reduction became more facile.

\subsection{Square wave voltammetry (SWV)}

In order to elucidate the unique electrochemical behaviour of FC/MWCNTs/CPE sensor towards FT detection, the electrochemical behaviour of $15 \mu \mathrm{M}$ FT was investigated at all three electrodes. Fig. 2D displays the square wave voltammograms for the $15 \mu \mathrm{M}$ FT on the CPE, MWCNTs/CPE and FC/MWCNTs/CPE. It shows that FC/MWCNTs/CPE exhibits a higher response in terms of peak current compared to the other electrodes. The enhanced peak current implies that electrochemical reduction of FT is more facile on FC/MWCNTs/CPE, which indicates that

Table 1 Chronocoulometry of $15 \mu \mathrm{M}$ FT for all three electrodes

\begin{tabular}{lll}
\hline Electrodes & $\begin{array}{l}\text { Diffusion coefficient } \\
\left(10^{-6} \mathrm{~cm}^{2} \mathrm{~s}^{-1}\right)\end{array}$ & $\begin{array}{l}\text { Surface coverage } \\
\left(10^{-10} \mathrm{~mole}^{-2}\right)\end{array}$ \\
\hline CPE & $6.23 \pm 0.07$ & 2.13 \\
MWCNTs/CPE & $6.21 \pm 0.05$ & 4.41 \\
FC/MWCNTs/CPE & $6.54 \pm 0.20$ & 10.12
\end{tabular}

proposed method is highly sensitive for the detection of FT. Performance of all three kinds of electrodes have been investigated statistically. For this purpose, square wave voltammograms for FT at 3 different CPEs, MWCNTs/CPEs and FC/ MWCNTs/CPEs have recorded and statistical parameters calculated (ESI Fig. S1-S4 and Table S1 $\dagger$ ). Fig. 3A reflects the electrocatalytic effect of FT at three different electrodes. Above findings enabled us to determine FT using SWV on FC/ MWCNTs/CPE sensor.

\subsection{Optimization of experimental conditions}

3.6.1 Supporting electrolytes and $\mathrm{pH}$ dependence study. The $\mathrm{pH}$ of the electrolyte solution has a significant influence on the electrochemical process of any electroactive molecules on the electrode surface. The influence of supporting electrolyte and $\mathrm{pH}$ of medium on the response of FT $(15 \mu \mathrm{M})$ was investigated using square wave voltammetry (SWV) employing FC/ MWCNTs/CPE. The effect of various supporting electrolytes on peak current and peak shape of FT was studied by employing various buffers viz.; Britton-Robinson $(\mathrm{pH}=2-10)$, acetate $(\mathrm{pH}$ $=4.8-6.4)$, borate $(\mathrm{pH}=3-10)$, phosphate $(\mathrm{pH}=3-11)$ and ammonia buffer $(\mathrm{pH}=7.5-11)$. Fig. 3B shows the effect of different supporting electrolytes on peak current and it can be noticed that better response in terms of peak shape and peak current was obtained in acetate buffer. From Fig. 3C it can be seen that maximum peak current and peak resolution was obtained at $4.5 \mathrm{pH}$. Thus, $\mathrm{pH} 4.5$ was employed for further studies. To prove the better performance of FC/MWCNTs/CPE in acetate buffer, 3 different FC/MWCNTs/CPEs have been place in each buffer and voltammograms were recorded. Statistical parameters for each buffers also calculated (ESI file S5-S8 \& Table S2 $\dagger$ ). It was noticed that the peak potentials of FT shifted towards more negative values with increase in $\mathrm{pH}$. The $\mathrm{pH}$ of the supporting electrolytes influences peak current and potential of FT indicating involvement of proton in the reduction reactions. The plot of $E_{\mathrm{p}} v s$. pH (Fig. 3D) was found to be linear with the slope of $54 \mathrm{mV} \mathrm{pH}^{-1}$ for FT which is closed to the expected value $59 \mathrm{mV}$ per $\mathrm{pH}$. This relationship can be described by following equation:

$$
E_{\mathrm{p}}(\mathrm{V})=-0.054 \mathrm{pH}-0.302\left(R^{2}=0.998\right)
$$



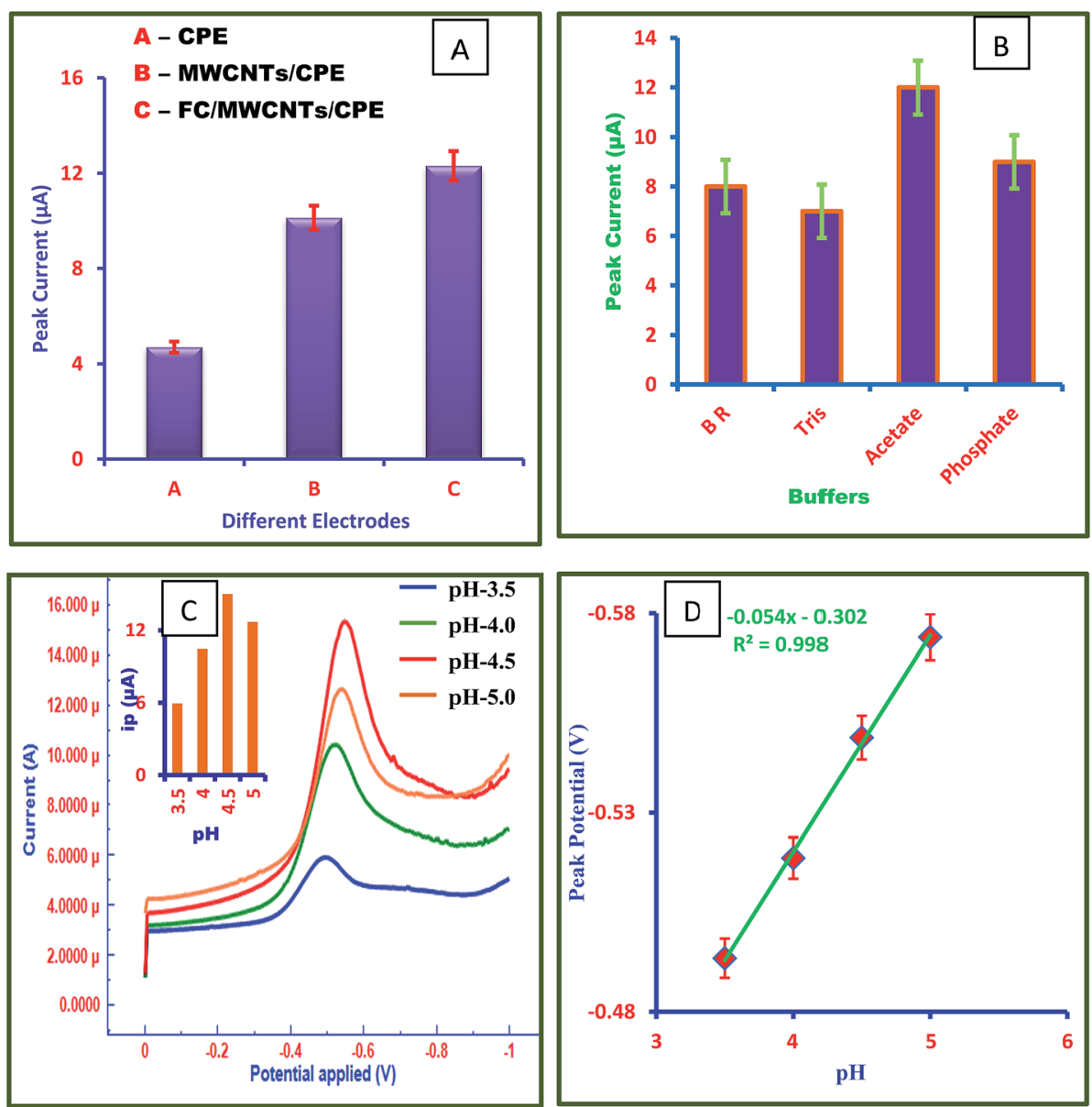

Fig. 3 (A) Comparison of electrocatalytic activity of all electrodes for FT detection; (B) a bar diagram for the effect of various supporting electrolytes employed for $15 \mu \mathrm{M} \mathrm{FT}$ at $\mathrm{pH} 4.5,(\mathrm{C})$ influence of $\mathrm{pH}$ on the peak current of $15 \mu \mathrm{M} \mathrm{FT}$ (inset: a bar diagram of the same), (D) a plot of peak potential $\left(E_{\mathrm{p}}\right)$ vs. $\mathrm{pH}$ for $15 \mu \mathrm{M} \mathrm{FT}$ ( $n=3$ every result/experiment was repeated three times).

Hence, it was evident that equal no of protons and electrons are involved in the reaction.

3.6.2 Effect of accumulation time and potential. Accumulation studies are simple and effective way to enhance the sensitivity of the electrode towards the analyte. Accumulation potential and time studies were carried out with $15 \mu \mathrm{M}$ FT at FC/ MWCNTs/CPE. Fig. 4A depicts the dependence of reduction peak currents of FT on the accumulation potential. The influence of the deposition potential on the peak currents of $15 \mu \mathrm{M}$ FT was investigated in the potential range of -1.0 to $1.0 \mathrm{~V}$. It can be seen from Fig. $4 \mathrm{~A}$ when the deposition potential was varied negatively from -1.0 to $0.1 \mathrm{~V}$, the peak currents increased. When more negative potential was applied, the peak current started decreasing. Therefore, a deposition potential of $0.1 \mathrm{~V}$ was chosen for further study. The influence of the deposition time on the anodic peak currents of $15 \mu \mathrm{M}$ FT was investigated at a fixed deposition potential of $0.1 \mathrm{~V}$ (Fig. 4B). Deposition time was the most important factor for determination of the detection limit in voltammetric analysis. When the deposition time varied in the range of $10 \mathrm{~s}$ to $160 \mathrm{~s}$, the peak current increased with the increase of deposition time. This was because at the same deposition potential, a longer deposition time would make FT be reduced more completely. Subsequently, it would lead to a higher peak current. However, when the deposition time was extremely long, the reduced FT covered the entire effective electrode surface, and the peak current started decreasing. Therefore, a deposition time of $120 \mathrm{~s}$ was chosen for further experiments.

\subsection{Calibration curve}

Fig. 4C shows the square wave voltammograms of FT at different concentrations in $0.2 \mathrm{M}$ acetate buffer at $\mathrm{pH} 4.5$ and Fig. 4D is the calibration plot between concentration of FT and peak current. The electrochemical responses of peak current of FT were increased linearly with its concentrations. The results indicated that the fabricated sensor is highly sensitive towards FT detection owing to the large peak current. The response of peak currents of FT at FC/MWCNTs/CPE was linear with the concentration of FT in the range of $0.1 \mu \mathrm{M}$ to $110 \mu \mathrm{M}$. Calibration curves for three different FC/MWCNTs/CPEs were also plotted (ESI file S9†).

\subsection{Validation of the developed method}

In order to validate the proposed analytical method with respect to parameters such as limit of quantification (LOQ), limit of 

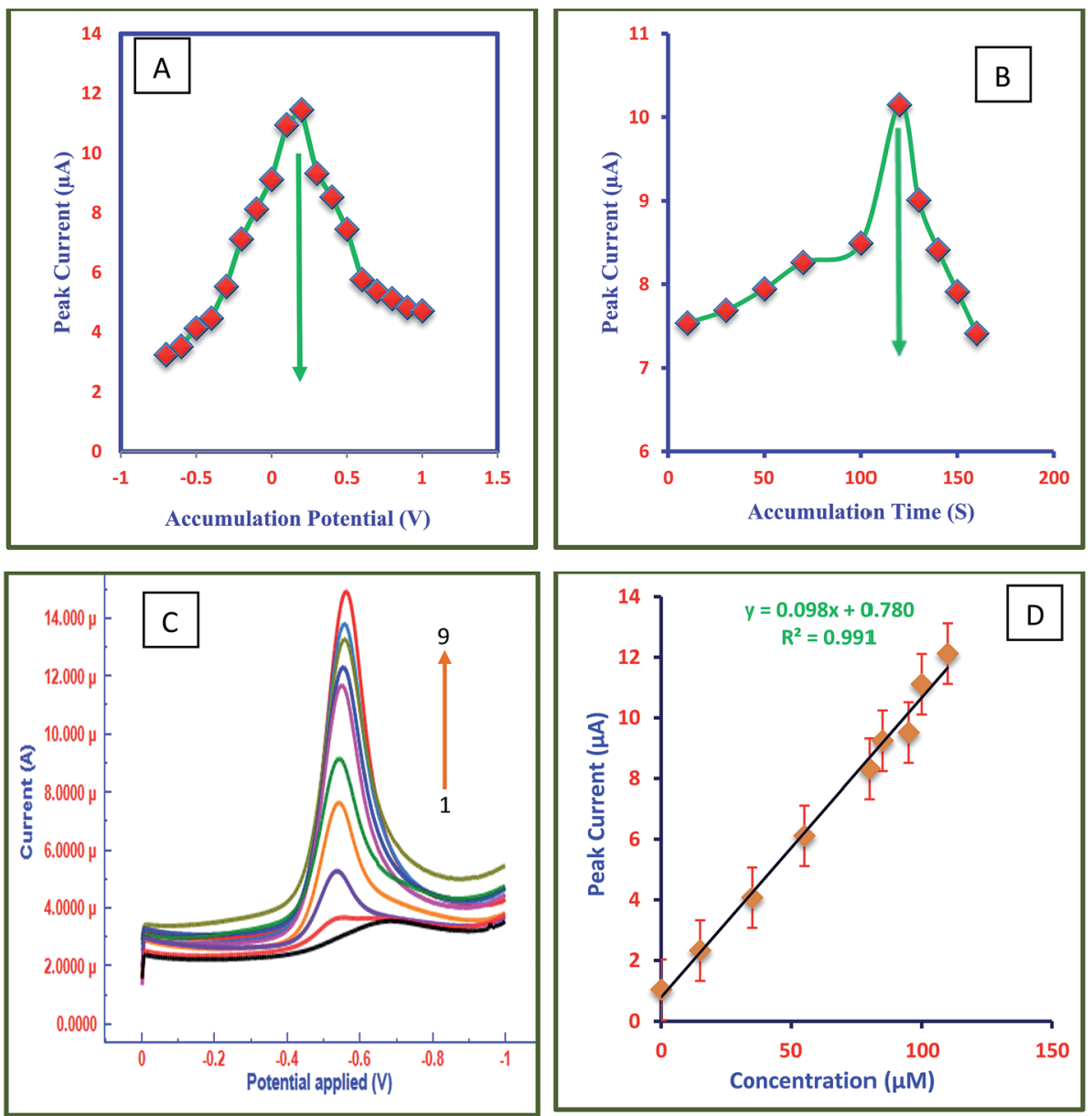

Fig. 4 (A) Influence of accumulation potential on the peak current of $15 \mu \mathrm{M} \mathrm{FT}$ on FC/MWCNTs/CPE (B) influence of accumulation time on the peak current of $15 \mu \mathrm{M}$ FT on FC/MWCNTs/CPE; (C) square wave voltammograms of FT at FC/MWCNTs/CPE containing different concentration of FT ranging from 0.1 to $110 \mu \mathrm{M}(1-9)$; (D) calibration plot of different concentration of drug and peak current.

detection (LOD), precision, accuracy, selectivity, recovery, robustness and ruggedness.

3.8.1 Detection and quantification limit. Detection limit was calculated by equation LOD $=3 s / \mathrm{m}$, where $s$ is standard deviation of intercept and $m$ is slope of the regression line. The calculated LOD value of FT is $0.001 \mu \mathrm{M}$. The quantification limit (LOQ) was examined by the equation LOQ $=10 \mathrm{~s} / \mathrm{m}$. The calculated LOQ value was $0.01 \mu \mathrm{M}$. Both LOD and LOQ values confirmed the sensitivity of the proposed methods.

3.8.2 Accuracy and precision. The accuracy of the proposed method was checked by performing five replicate measurements for $15 \mu \mathrm{M}$ FT over a single day (intra-day assay, $n$ $=5$ ) and after five days (inter-day assay, $n=5$ ) at the fabricated electrode. The analytical precision of the developed method was verified from the reproducibility of 5 determinations of $15 \mu \mathrm{M}$ FT and the estimated relative standard deviation (RSD\%) was $1.8 \%$. The recovery tests were carried out by standard addition method and results are depicted in Tables 2 and $\mathrm{S} 3 . \dagger$

3.8.3 Stability of FC/MWCNTs/CPE. In order to investigate the stability of the fabricated sensor, the sensor was placed in pH 4.5 acetate buffer containing $15 \mu \mathrm{M}$ FT and voltammograms were recorded after an interval of 5 days. It was noticed that peak current decreased by $2.1 \%$, which showed that the sensor had good stability. The sensor was found to retain $98.6 \%$ of its initial peak current response for a FT concentration of $15 \mu \mathrm{M}$ FT by the end of 30 days, which shows the long-term storage stability of electrode during the determinations in aqueous solutions. The results indicate a good stability of the sensor and capacity for repeated measurement to be performed on the same sensor.

3.8.4 Specificity/selectivity. Specificity is the ability of the method to measure the analyte response in the presence of all of the potential impurities. The selectivity of the optimized

Table 2 Precision and bias of assay for standard FT solution by the proposed voltammetric procedure $(n=5)$

\begin{tabular}{lllll}
\hline Method & $\begin{array}{l}\text { Concentration } \\
\text { taken }(\mu \mathrm{M})\end{array}$ & $\begin{array}{l}\text { Concentration } \\
\text { found }(\mu \mathrm{M})\end{array}$ & $\begin{array}{l}\text { Recovery }(\%) \\
(n=5)\end{array}$ & $\begin{array}{l}\text { Precision \% } \\
\text { RSD }(n=5)\end{array}$ \\
\hline SWV & $\begin{array}{l}\text { Intra-day } \\
15\end{array}$ & 14.95 & 99.66 & 1.24 \\
& $\begin{array}{l}\text { Inter-day } \\
15\end{array}$ & 14.75 & 98.33 & 3.14
\end{tabular}


procedure for determination of FT was examined in the presence of foreign species such as ascorbic acid, uric acid, glucose, valine, $\mathrm{Ca}^{2+}, \mathrm{Mg}^{2+}$, tryptophan, tyrosine and dopamine. Samples containing $15 \mu \mathrm{M}$ FT and different concentrations of the excipient under evaluation were analyzed by means of the proposed method. The obtained mean percentage recoveries and $\mathrm{RSD} \%$ values based on an average of five replicate measurements, $98.64 \pm 0.60$ to $100.8 \pm 0.50$, showed no

Table 3 Comparison between various electroanalytical methods for the determination of FT with the proposed methods

\begin{tabular}{|c|c|c|c|c|}
\hline S. no. & Modified electrode & $\begin{array}{l}\text { Linearity } \\
\text { range }(\mu \mathrm{M})\end{array}$ & $\begin{array}{l}\text { Limit of } \\
\text { detection } \\
(\mu \mathrm{M})\end{array}$ & Ref. \\
\hline 1 & CPE in surfactant media & $72.4-724$ & 0.181 & 44 \\
\hline 2 & AgNPs modified GCE & $10-1000$ & 9.33 & 45 \\
\hline 3 & Pencil graphite electrodes & $0.398-6.36$ & 0.0155 & 47 \\
\hline 4 & DME (polarography) & $1-1000$ & - & 48 \\
\hline 5 & FC/MWCNTs/CPE & 0.1-110 & 0.001 & $\begin{array}{l}\text { Present } \\
\text { work }\end{array}$ \\
\hline
\end{tabular}

significant interference from excipients. Interference study has also been carried out in the presence of more structurally relevant molecules such as nilutamide. It was observed that fabricated electrode cannot be used to determine both the drugs simultaneously. However, separately nilutamide can be analysed by the fabricated electrode. Thus, the proposed procedure can be considered to be specific.

3.8.5 Robustness. The robustness was examined by evaluating the influence of small variations of some of the most important procedure variables, including preconcentration potential and time and $\mathrm{pH}$. The obtained results provided an indication of the reliability of the proposed procedure for the assay of FT; hence, it can be considered as robust. The obtained mean percentage recoveries based on an average of five replicate measurements were not significantly affected within the studied range of variations of some operational parameters, and consequently the proposed procedure can be considered as robust.

3.8.6 Ruggedness. The ruggedness of the measurements is defined as the degree of reproducibility of results obtained by analysis of same sample under variety of normal test conditions
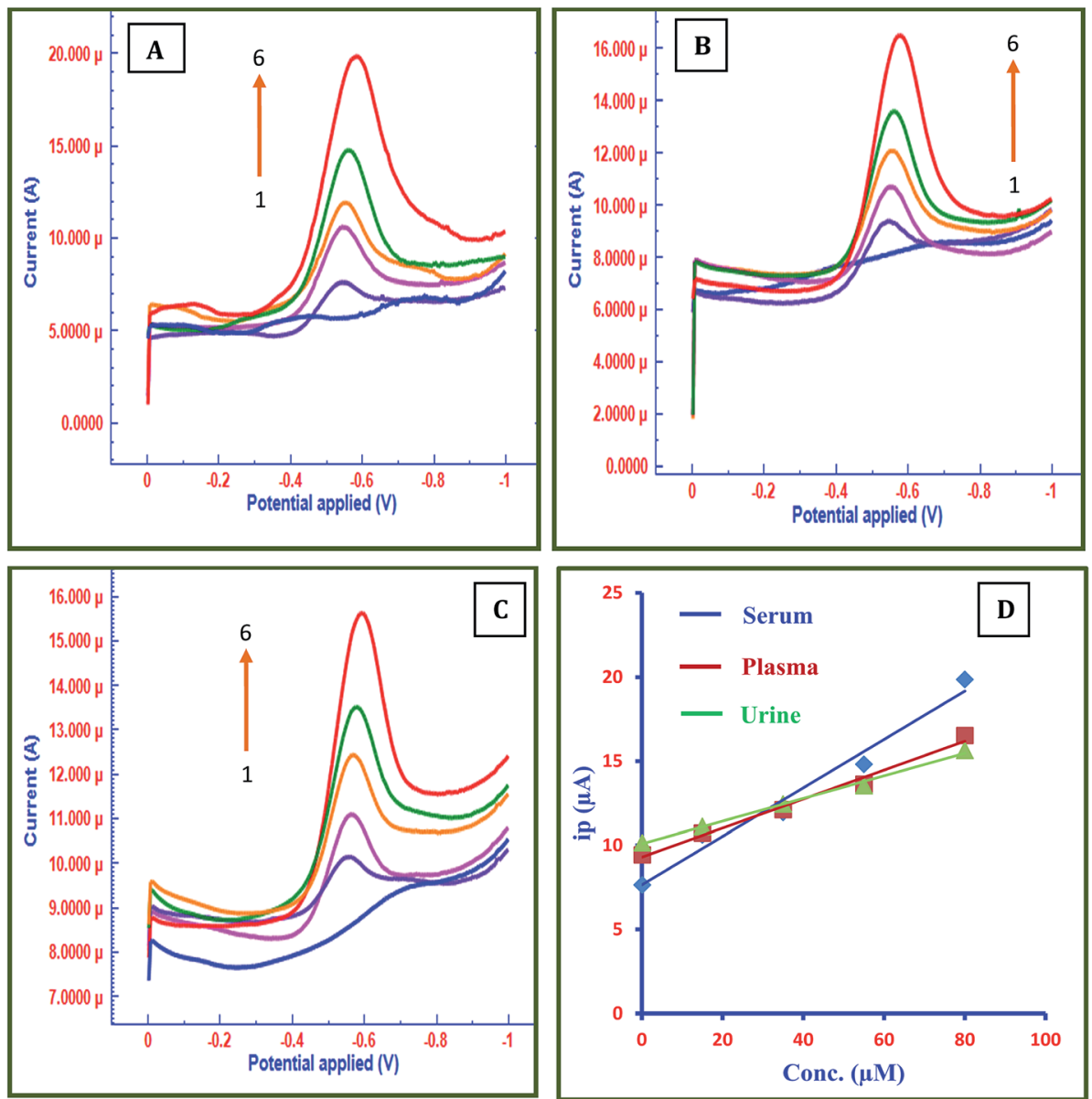

Fig. 5 (A) SWV of different concentration of spiked human serum sample at fabricated sensor, (1) serum, (2) 0.1 , (3) 15 , (4) 35 , (5) 55 , (6) $80 \mu \mathrm{M}$; (B) SWV of different concentration of spiked human plasma sample at fabricated sensor, (1) plasma, (2) 0.1 , (3) 15 , (4) 35, (5) 55, (6) $80 \mu \mathrm{M}$; (C) SWV of different concentration of spiked human urine sample at fabricated sensor, (1) urine, (2) 0.1, (3) 15 , (4) 35 , (5) 55 , (6) $80 \mu \mathrm{M}$; (D) calibration plots of peak current and different concentration of FT in serum, plasma and urine samples. 
Table 4 Determination of FT drug in human serum, plasma and urine using fabricated sensor

\begin{tabular}{lllll}
\hline Samples & $\begin{array}{l}\text { Regression equation } I_{\mathrm{p}} \\
(\mu \mathrm{A})\end{array}$ & $\begin{array}{l}\text { Linearity } \\
(\mu \mathrm{M})\end{array}$ & $R$ & $\begin{array}{l}\text { LOD } \\
(\mu \mathrm{M})\end{array}$ \\
\hline Serum & $I_{\mathrm{p}}=28.60 x+4.399$ & $0.1-80$ & 0.954 & 0.007 \\
Plasma & $I_{\mathrm{p}}=17.09 x+7.358$ & $0.1-80$ & 0.967 & 0.009 \\
Urine & $I_{\mathrm{p}}=13.38 x+8.560$ & $0.1-80$ & 0.979 & 0.004
\end{tabular}

such as different laboratories and different lot of reagents, under the same operational conditions at different elapsed time by two different analysts. The methods were found to be rugged with the results of variation coefficients $1.5 \%$ and $2.5 \%$ for SWV method for first and second analysts, respectively. The results show no statistical differences between different analysts.

\subsection{Comparison of proposed method with literature methods}

A comparison between the analytical performances of the proposed method with previously reported methods for determination is shown in Table 3. The data reveals that the FC/ MWCNTs/CPE shows superior analytical performance in terms of very low detection limit, wide linear dynamic range, high sensitivity, good reproducibility, and repeatability over methods reported in literature. In addition present method is simple, and does not involve any pre-treatment step.

\subsection{Analytical applications}

Analytical applications of the fabricated sensor were explored in the determination of FT in real samples by testing them on human blood serum, plasma and urine samples. The serum, plasma and urine samples were analyzed at FC/MWCNTs/CPE using calibration method. Serum, plasma and urine samples were diluted with $0.2 \mathrm{M}$ acetate buffer of $\mathrm{pH} 4.5$ without any pretreatment. Fig. 5 shows the SWVs in serum (A), plasma (B) and urine (C). Fig. 5D depicts calibration plots of all three samples. The samples were spiked with known amounts of FT and its voltammetric responses were recorded. Good linearity and LOD were obtained as shown in Table 4 providing further evidence that this is a reliable method that can be applied to the determination of FT in real samples such as serum, plasma and urine. This also suggests the accuracy and selectivity of the developed method.

\section{Conclusion}

A highly enhancing sensing platform based on ferrocene and MWCNTs fabricated electrochemical sensor FC/MWCNTs/CPE was established for the trace detection of flutamide. The fabricated sensor showed an excellent electrocatalytic activity in increasing the cathodic over-potential and remarkable enhancement of cathodic current of FT compared with electrochemical performances obtained at CPE and MWCNTs/CPE. This selective and sensitive platform is used for the determination of flutamide for the first time. Electrochemical behaviour of FT at all three electrodes namely CPE, MWCNTs/ CPE and FC/MWCNTs/CPE has been evaluated. It was noticed that FC/MWCNTs/CPE exhibited excellent selectivity and sensitivity towards FT detection. Moreover, the proposed method is highly sensitive having very low detection limit, wide linear dynamic range, very good reproducibility, and repeatability over other methods reported in literature. It is expected that the proposed method will be of great help to both clinical as well as pharmaceutical industries.

\section{Acknowledgements}

The authors are thankful to the Head, Department of Chemistry, K L University, Guntur, Andhra Pradesh, India for providing necessary laboratory facilities. This work is financially supported by Science and Engineering Research Board, (Grant No. SB/FT/ CS-019/2014) New Delhi, India, under its Fastrack Young Scientists Scheme and UGC, New Delhi under its UGC research award scheme for teachers (Grant no. 30-12/2015 SA-II).

\section{References}

1 R. N. Brogden and S. P. Clissold, Flutamide Drug., 1989, 38, 185.

2 M. Jonler, M. Riehmann and R. C. Bruskewitz, Drugs, 1994, 47, 66.

3 A. K. Jain, V. K. Gupta, L. P. Singh and J. R. Raisoni, Electrochim. Acta, 2006, 51, 2547.

4 V. K. Gupta, M. R. Ganjali, P. Norouzi, H. Khani, A. Nayak and S. Agarwal, Crit. Rev. Anal. Chem., 2011, 41, 282.

5 R. Jain, V. K. Gupta, N. Jadon and K. Radhapyari, Anal. Biochem., 2010, 407, 79.

6 V. K. Gupta, A. K. Singh, S. Mehtab and B. Gupta, Anal. Chim. Acta, 2006, 566, 5.

7 A. Kutluay and M. Aslanoglu, Anal. Chim. Acta, 2014, 839, 59.

8 P. K. Kalambate, B. J. Sanghavi, S. P. Karna and A. K. Srivastava, Sens. Actuators, B, 2015, 213, 285.

9 T. Tavana, M. A. Khalilzadeh, H. Karimi-Maleh, A. A. Ensafi, H. Beitollahi and D. Zareyee, J. Mol. Liq., 2012, 168, 69.

10 Y. Teng, L. Fan, Y. Dai, M. Zhong, X. Lu and X. Kan, Biosens. Bioelectron., 2015, 71, 137.

11 J. A. Rather and K. D. Wael, Sens. Actuators, B, 2012, 171, 907. 12 R. Khan and M. Dhayal, Biosen. Bioelectron., 2009, 24, 1700.

13 B. J. Sanghavi and A. K. Srivastava, Anal. Chim. Acta, 2011, 706, 246.

14 B. J. Sanghavi and A. K. Srivastava, Electrochim. Acta, 2010, 55, 8638.

15 R. R. Gaichore and A. K. Srivastava, J. Appl. Electrochem., 2012, 42, 979.

16 P. K. Brahman, R. A. Dar and K. S. Pitre, Sens. Actuators, B, 2013, 176, 307.

17 P. K. Brahman, R. A. Dar and K. S. Pitre, Sens. Actuators, B, 2013, 176, 307.

18 P. K. Brahman, N. Pandey, S. N. Topkaya and R. Singhai, Talanta, 2015, 134, 554.

19 M. Siswana, K. I. Ozoemena and T. Nyokong, Talanta, 2006, 69, 1136. 
20 R. N. Goyal, V. K. Gupta and S. Chatterjee, Biosen. Bioelectron., 2009, 24, 1649.

21 J. A. Rather and K. D. Wael, Sens. Actuators, B, 2013, 176, 110. 22 L. Qu and L. Dai, J. Am. Chem. Soc., 2005, 127, 10806.

23 S. Alwarappan, S. Prabhulkar, A. Durygin and C.-Z. Li, J. Nanosci. Nanotechnol., 2009, 9, 2991.

24 G. Wu, Y. S. Chen and B. Q. Xu, Electrochem. Commun., 2005, 7, 1237.

25 S. Iijima, Nature, 1991, 354, 56.

26 H. Qi, C. Zhang and X. Li, Sens. Actuators, B, 2006, 114, 364.

27 J. Weber, A. Kumar, A. Kumar and S. Bhansali, Sens. Actuators, B, 2006, 117, 308.

28 N. Sato and H. Okuma, Sens. Actuators, B, 2008, 129, 188.

29 Y. C. Tsai and C. C. Chiu, Sens. Actuators, B, 2007, 125, 10.

30 Y. Lin, R. X. Wang and X. M. Li, Electrochem. Commun., 2005, 7, 597.

31 S. Hrapovic, Y. Liu and K. B. Male, Anal. Chem., 2004, 76, 1083.

32 Y. C. Tsai, S. C. Li and J. M. Chen, Langmuir, 2005, 21, 3653.

33 M. G. Zhang, A. Smith and W. Gorski, Anal. Chem., 2004, 76, 5045.

34 I. Svancara, K. Kalcher, A. Walcarius and K. Vytras, Electroanalysis with Carbon Paste Electrodes, CRC Press, Francis \& Taylor, 2012.

35 A. Leniart, M. Brycht, B. Burnat and S. Skrzypek, Sens. Actuators, B, 2016, 231, 54.
36 J. Robak, B. Burnat, A. Leniart, A. Kisielewska, M. Brycht and S. Skrzypek, Sens. Actuators, B, 2016, 236, 318.

37 G. Wang, J. J. Xu, L. H. Ye, J. J. Zhu and H. Y. Chen, Bioelectrochem., 2002, 57, 33.

38 J. Lin, C. He, Y. Zhao and S. Zhang, Sens. Actuators, B, 2009, 137, 768.

39 Y. Wang, W. Z. Wei, J. X. Zeng, X. Y. Liu and X. D. Zeng, Microchim. Acta, 2008, 160, 253.

40 L. C. Jiang and W. D. Zhang, Biosens. Bioelectron., 2010, 25, 1402.

41 D. Farthing, D. Sica, I. Fakhry, D. L. Walters, E. A. Cefali and G. Allan, Biomed. Chromatogr., 1994, 8, 251.

42 P. Nagaraja, K. R. Sunitha and M. F. Silwadi, J. Pharm. Biomed. Anal., 2000, 23, 617.

43 P. Nagaraja, H. R. Arun Kumar, R. A. Vasantha and H. S. Yathirajan, Int. J. Pharm., 2002, 235, 113.

44 P. K. Brahman, R. A. Dar, S. Tiwari and K. S. Pitre, Colloids Surf., A, 2012, 396, 8.

45 F. Ahmadi, J. B. Raoof, R. Ojani, M. Baghayeri, M. M. Lakouraj and H. Tashakkorian, Chin. J. Catal., 2015, 36, 439.

46 Y. Li, P. Wang, F. Li, X. Huang, L. Wang and X. Lin, Talanta, 2008, 77, 833.

47 Y. M. Temerk, H. S. M. Ibrahim and W. Schuhmann, Electroanalysis, 2016, 28, 372.

48 A. Alvarez-Lueje, C. Pena, L. J. Nunez-Vergara and J. A. Squella, Electroanalysis, 1998, 10, 1043. 